Original article

\title{
Pathomorphological distinction between Er:YAG and diode lasers on the excisional biopsy of the oral mucosa
}

\author{
Georgi T. Tomov, Svitlana Y. Bachurska, Desislava A. Tashkova, Georgi P. Ivanov \\ Medical University of Plovdiv, Plovdiv, Bulgaria
}

Received 18 January 2013, Accepted 30 January 2013.

(C) 2013, Tomov G.T., Bachurska S.Y., Tashkova D.A., Ivanov G.P.

(C) 2013, Russian Open Medical Journal

Abstract: Background - In oral pathology, laser devices can provide important advantages, especially in the treatment of certain lesions. However, there is controversy about the use of some wavelengths in the analysis of suspected dysplastic or neoplastic lesions, raising doubt about the laser's suitability for use in biopsy procedures. The present pilot study evaluates the pathomorphological characteristics and suitability of Er:YAG and diode lasers for performing excisional biopsies in the oral mucosa with special emphasis on the extent of the thermal damage zone created.

Material and Methods - 10 patients were randomly assigned to one diode or one Er:YAG laser groups. The Er:YAG laser(2940nm) was used in a pulsed wave mode $200 \mathrm{~mJ} / 35 \mathrm{~Hz}$ with a power of $7 \mathrm{~W}$. Power settings for the diode laser $(810 \mathrm{~nm})$ were $3 \mathrm{~W}$ in a pulsed mode. The thermal damage zone of the two lasers and intraoperative and postoperative complications were assessed and compared.All biopsy specimens were evaluated by optical microscopy by threeblinded pathologists. After establishing the pathomorphological diagnosis of the lesion assessed, the pathologists measured the maximal width of the peripheral thermal damage zone in the specimen in $\mu \mathrm{m}$ and classified it using appropriate index.

Results - The peripheral thermal damage zone on the borders of the excisional biopsies was significantly smaller with the Er:YAG laser compared to the diode laser regarding values in $\mu \mathrm{m}$ or pathomorphological index scores. No postoperative complications occurred in any of the two groups.

Conclusions - The Er:YAG laser seems to be appropriate for excisional biopsies of benign oral mucosal lesions. The Er:YAG laser offers clear advantages in terms of smaller thermal damage zones over the diode laser. Although in some samples thermal damage was minimally visible, in all samples histological evaluation was clearly possible. The study demonstrated that the Er:YAG laser can be safely used in oral biopsy investigations while ensuring a successful histological evaluation, which is fundamental to correct clinical management.

Keywords: Er:YAG laser, diode laser, excisional biopsy

Cite as Tomov GT, Bachurska SY, Tashkova DA, Ivanov GP. Pathomorphological distinction between Er:YAG and diode lasers on the excisional biopsy of the oral mucosa. Russian Open Medical Journal 2013; 2: 0107.

Correspondence to Dr Georgi T. Tomov. Address: 3 Hristo Botev Blv, 4000 Plovdiv, Bulgaria, Oral Pathology Department, Faculty of Dental Medicine, Medical University - Plovdiv Phone: +359 896742065 E-mail: dr.g.tomov@gmail.com

\section{Introduction}

Since the first laser system was developed in the 1960s it has been widely used in different areas of medicine such as ophthalmology, dermatology and general surgery. In the beginning laser devices used in dentistry were focused only on hard tissues. Although more than twenty years of experiments and investigations have established the capability of the laser systems to treat a great variety of pathological changes in the soft tissues of the oral cavity [1]. Today lasers are often used in dentist practices because of some their undeniable advantages: lack of bacterial contamination, strong hemostatical effect, postoperative reduction of the inflamation and serious decrease of pain levels amongst the patients $[2,3]$.

Currently more than 10 different types of the lasers are available for use in the field of dentistry. They can be classified by wavelength, power and kind of tissue which they impact on. However, the characteristic which bands together all types of devices, ignoring the different mechanisms of action, is the ability to change light energy into the heat.This can cause irreversable thermal damage of the treated tissue [4].

Er:YAG was developed less than 15 years ago with the aim of managing lesions occurring in all kinds of tissues in the oral cavity like mucosa, bone, enamel, dentine and cement $[5,6]$. The wavelength $(2940 \mathrm{~nm})$ that the laser produced is well absorbed by water molecules, which leads to their vaporization, therefore the treated tissue is removed with a proven bactericidal effect [7].

Diode laser (with wavelength 810 and $980 \mathrm{~nm}$ ) is used in dentistry for desinfection, for bleaching teeth and in oral surgery for soft tissue applications. The wavelength that the laser produces is well absorbed by hemoglobin molecules in soft tissues which produces a potential risk of deep thermal damage [2].

Obviously, the use of lasers in dental practices as a biopsy tool has a risk of damaging the biopsy specimens. This could make histological evaluation extremely difficult, especially when the 
condition of the resection zone should be analyzed in order to discover any possible neoplastic infiltration. Accordingly this makes us believe in a nessecity to comprehensively investigate which laser system is more sutable for accurate biopsy performance without any unfavourable effects on the treated tissues.

The purpose of the present study is to compare certain pathomorphological characteristics of the tissue from the oral mucosa removed as an excisional biopsy performed by Er:YAG and diode lasers, focusing on the levels of the thermal damage of the specimen's peripheral zone.

\section{Materials and methods}

The study was performed on ten patients referred for treatment to the Oral Pathology division, Faculty of Dental Medicine, Plovdiv, Bulgaria. Necessary precautions and safety protocols were undertaken by the operator and the patient all through the operating procedure. Five biopsy specimens were obtained from suspected benign lesions on the oral mucosa using Er:YAG laser (2940 nm, LiteTouch, Syneron, Israel) (Figure 1), and five others using diode laser ( $810 \mathrm{~nm}$, D-Touch, Syneron, Israel) (Figure 2). The Er:YAG laser was used in a pulsed wave mode $200 \mathrm{~mJ} / 35 \mathrm{~Hz}$ with a power of $7 \mathrm{~W}$. Power setting for the diode laser was $3 \mathrm{~W}$ in a pulsed mode. All the surgical excisions were performed by the same operator (G.T.). Biopsy specimens were sent to the Department of General and Clinic Pathology for histological evaluation.

\section{Pathomorphological performance and examination}

Following the excision, the specimens were placed in $10 \%$ formalin solution for adequate fixation. The speciment were embedded in parafin and $5 \mu \mathrm{m}$ sections were prepared. The hematoxylin-eosin stained sections were evaluated separately by three different pathologists. The light microscope Olympys $\mathrm{CH} 30$ on $4 x n 10 x$ magnifications and the macromethric system Objektmikrometer и Okularmikrometer of Carl-Zeiss Jena were used for pathomorphological examination. The sections without artefacts were evaluated for further histological features:

a) Epithelial changes: nuclear, cytoplasmic and cell membrane.

b) Connective tissue changes: thermal necrosis, collagen denaturation and tissue alteration.

c) Presens or absence of vascular modifications: thrombosis or stasis.

d) All changes observed (expressed in micrometers and evaluated separately for the epithelium, connective tissue and vassel modifications) were considered to be a thermal damage zone and the widest one $(1050 \mu \mathrm{m})$ was chosen as a main value. The same value was divided into the three equal parts indicating, respectively, zones with mild $(0-350 \mu \mathrm{m})$, moderate $(351-700 \mu \mathrm{m})$ and severe $(701-1050 \mu \mathrm{m})$ thermal damage.

\section{Results}

The biopsy specimens, without any exception, performed using Er:YAG laser are in the zone with the mild thermal damage (Figures 3a, 3b and Figures 4a, 4b).

Pathomorphological changes obtained by the diode lazer are in the zones of the moderate and severe thermal damage (Figures $5 \mathrm{a}$ and $5 b)$.
Destribution of the specimens from both groups due to the level of the thermal damageis presented on the Table 1.

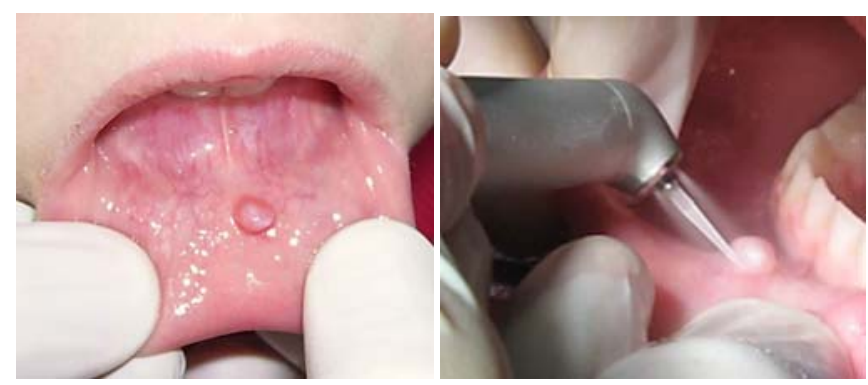

Figure 1. Excisional biopsy using Er:YAG laser

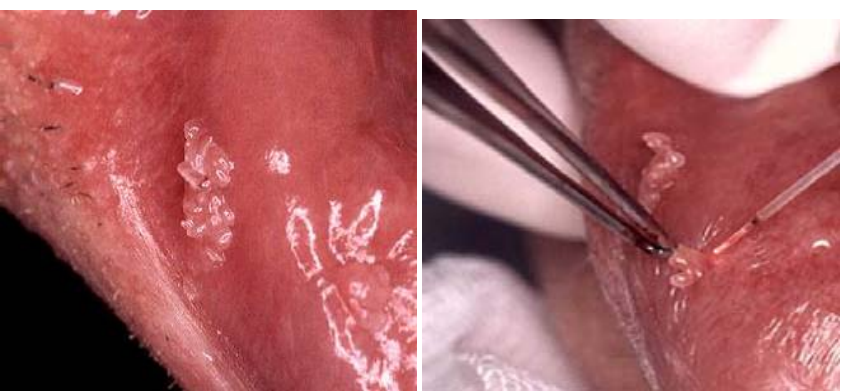

Figure 2. Excisional biopsy using diode laser

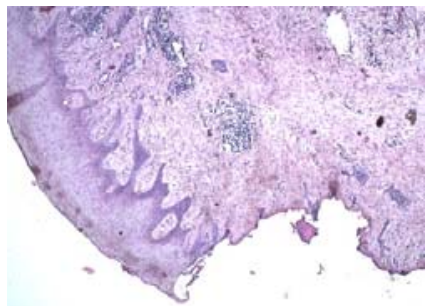

a

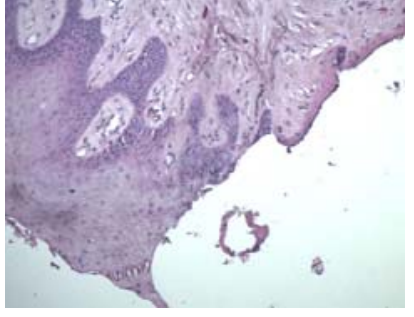

Figure 3. a) Magnificance $x 4$. Biopsy sample performed using Er:YAG laser and diagnosed as lichen planus; b) Magnificance x10. Peripheral zone of the speciment shows limited changes coused by thermal effect of the laser.

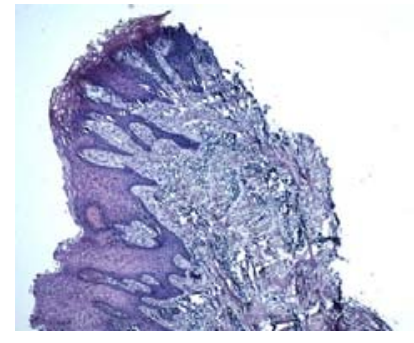

a

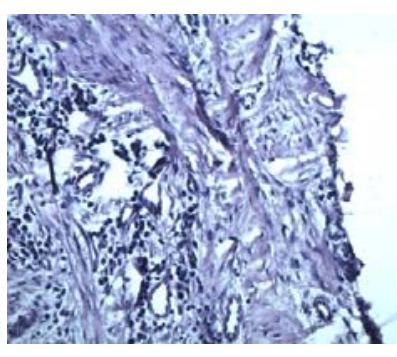

b
Figure 4. a) Magnificance x4. Biopsy sample performed using Er:YAG laser and diagnosed as piogenic granuloma; b) Magnificance x20. Peripheral zone of the speciment shows limited changes coused by thermal effect of the laser. 
Changes in the particular histological structures (epithelium, connective tissue and vessels) are demonstrated on the Figure 6.

The results date were reconfirmed by statistical platform SPSS.

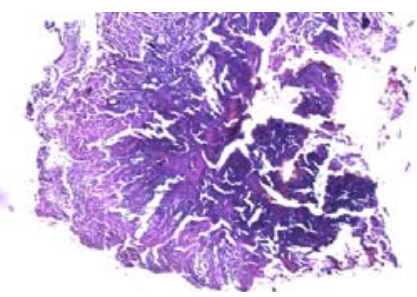

a

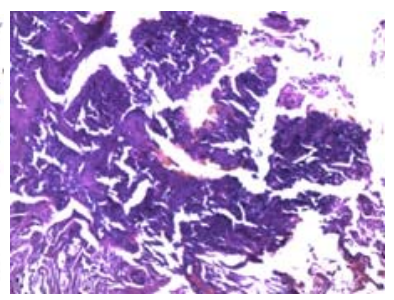

b
Figure 5. a) Magnificance x4. Biopsy sample performed using diode laser. Pathomorphological diagnosting is impossible because of the small size of the speciment and severe thermal damage couse by the diode laser; b) Magnificance x20. Peripheral zone of the speciment shows sever changes as protein denaturation and completely lost of the nuclei coused by the diode laser.

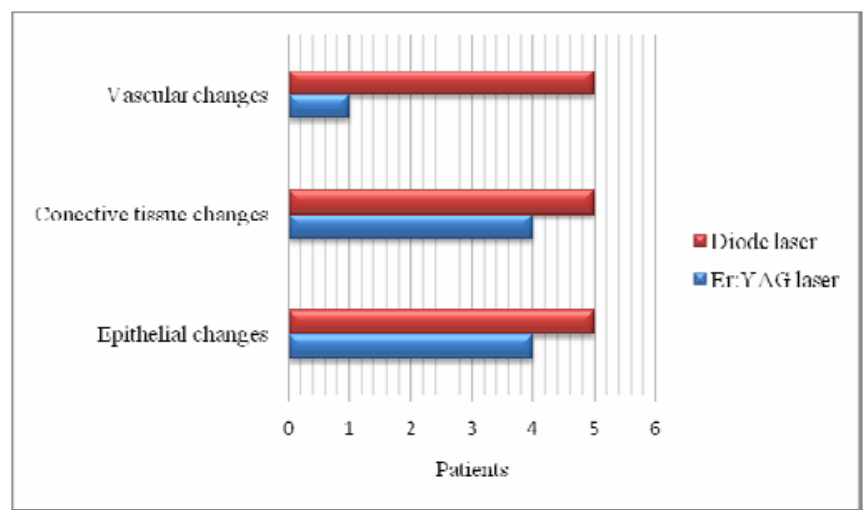

Figure 6. Changes in the particular histological structures in both groups (Er:YAG laser and diode laser).

Table 1. Disociation of the specimens ( $n-10)$ due to the level of the zone with thermal damage in Er:YAG and diode lasers groups

\begin{tabular}{cccc}
\hline Damage level & mild & moderate & severe \\
\hline Er:YAG laserspecimens & 5 & 0 & 0 \\
diode laserspecimens & 0 & 1 & 4 \\
\hline
\end{tabular}

\section{Discussion}

The level of thermal damage coused by Er:YAG laser does not impact on the capability of the histological examination of the specimen's peripheral zone. This corelates with the results of the other researchers investigating laser systems effects on the soft tissue in the oral cavity $[8,9]$. The advantage of the Er:YAG laser is a weeker penetration of the laser energy into the tissue $(0.05 \mathrm{~mm})$ and a presence of additional water cooling.

Pathomorphological changes obtained by the diode lazer are in the zones of the moderate and severe thermal damage. This level of modification makes difficult or even impossible to accurately evaluate cells' changes in the treated areas. There are different speculations about the diode laser thermal effects on the soft tissue in the oral cavity. Some authors report tissue modifications not influencing pathomorphological examination $[2,11]$, whereas others have results comparable with ours $[3,10]$. Results probably vary depending on what research method the group of investigators had chosen.

\section{Conclusions}

Three parameteres are important for the evaluation of new technologies used for excisional biopsy in oral surgery: a) patient compliance during procedure, b) convenience for the surgeon, and c) quality of the biopsy specemen [11]. The analysis of the literature shows that laser systems support accurate excision with minimal short- and long-term morbidity [12].

The Er:YAG laser has a less negative thermal effect on the soft tissue in the oral cavity compared with the diode laser. This allows precise histological examination despite the thermal damage of the peripheral zone, which makes this laser system extremely suitable for the oral mucosa excisional biopsy performance.

Conflict of interest: none declared.

\section{Reference}

1. Rizoiu IM, Eversole LR, Kimmel Al. Effects of an erbium, chromium: yttrium, scandium, gallium, garnet laser on mucocutanous soft tissues. Oral Surg Oral Med Oral Pathol Oral RadiolEndod 1996; 82: 386-95. (PMID: 8899775)

2. Pick RM, Colvard MD. Current status of lasers in soft tissue dental surgery. J Periodontol 1993; 64: 589-602. (PMID: 8366410)

3. Goharkhay K, Moritz A, Wilder-Smith P, Schoop U, Kluger W, Jakolitsch $S$, Sperr W. Effects on oral soft tissue produced by a diode laser in vitro. Lasers Surg Med 1999; 25: 401-6. (PMID: 10602132) (doi: 10.1002/(SICI)1096-9101(1999)25:5<401::AID-LSM6>3.0.CO;2-U)

4. Romeo U, Palaia G, Del Vecchio A, Tenore G, Gambarini G, Gutknecht $N$, De Luca M. Effects of KTP laser on oral soft tisuues. An in vitro study. Lasers Med Sci 2010; 25: 539-43. (PMID: 20162316) (doi: 10.1007/s10103-010-0756-2)

5. Eversole LR, Rizoiu IM. Preliminary investigations on the utility of an erbium, chromium YSGG laser. J Calif Dent Assoc 1995; 23: 41-7. (PMID: 9052027)

6. Tsanova Sn, Tomov G. Morphological changes in hard dental tissues prepared by Er:YAG laser, Carisolv and rotary instruments. A scanning electron microscopy evaluation. Folia Medica 2010; 52(3): 46-55. (PMID: 21053673) (doi: 10.2478/v10153-010-0006-1)

7. Lubart R, Kesler G, Lavie R, Friedmann H. Er;YAG laser promotes gingival wound repair by photo-dissociating water moleculs. Photomed Laser Surg 2005; 23: 369-72. (PMID: 16144478)

8. Vescovi P, Corcione L, Meleti M, Merigo E, Fornaini C, Manfredi M, et al. Nd:YAGlaset versus traditional scalpel. A preliminary histological analysis of speciments from the human oral mucosa. Lasers Med Sci 2010; 25: 685-91. (doi: 10.1007/s10103-010-0770-4) (PMID: 20393771)

9. Belcheva A, Tomov G, Petrova S, Tsvetanov T. Multidisciplinary approach in excisional biopsy papilloma removal with Lite Touch Er:YAG laser in pediatric dental patient. Int J Pediatr Dent 2011; 21(3): 155.

10. Cercadillo-lbarguren I, España-Tost A, Arnabat-Domínguez J, Valmaseda-Castellón E, Berini-Aytés L, Gay-Escoda C. Histologic evaluation of termal damage produced on soft tissues by $\mathrm{CO} 2$, Er,Cr:YSGG and diode lasers. Med Oral Patol Oral Cir Bucal 2010; 15(6): e912-8. (PMID: 20526250) (doi: 10.4317/medoral.15.e912)

11. Fornaini C, Rocca JP, Bertrand MF, Merigo E, Nammour S, Vescovi P. $\mathrm{Nd}$ :YAG and diode laser in the surgical management of soft tissues related to orthodontic treatment. Photomed and Laser Surg 2009; 25(5): 381-392. (PMID: 17975951) (doi: 10.1089/pho.2006.2068)

12. Gutkneht N. Proceedings of the 1st international workshop of Evidensed-Based Dentistry on laser in dentistry. Quintessence Publishing Co Ltd, Chicago, USA, 2007. 
Authors:

Georgi T. Tomov - DDS, MSc, PhD, Associate Professor, Division of oral pathology, Faculty of Dental Medicine, Medical University of Plovdiv, Plovdiv, Bulgaria;

Svitlana Y.Bachurska - MD, Assistant, Department of General and Clinical Pathology, Faculty of Medicine, Medical University of Plovdiv, Plovdiv, Bulgaria;

Desislava A. Tashkova - MD, Assistant, Department of General and Clinical Pathology, Faculty of Medicine, Medical University of Plovdiv, Plovdiv, Bulgaria;

Georgi P. Ivanov - MD, MSc, Senior Assistant, Department of General and Clinical Pathology, Faculty of Medicine, Medical University of Plovdiv, Plovdiv, Bulgaria. 